\title{
Retention of maze learning following paradoxical sleep deprivation in rats*
}

\author{
T. L. HOLDSTOCK and G. J. VERSCHOOR \\ University of the Witwatersrand, Johannesburg, South Africa
}

\begin{abstract}
In an attempt to evaluate the importance of paradoxical sleep in memory consolidation, rats were maintained on inverted flower pots for 3 days after learning in two T-maze experiments. Living on flower pots with large and small diameters did not affect retention of food-motivated position habits any differently than did home cage living. The possibility of an interactive effect between paradoxical sleep deprivation and the nature of the learning task was suggested as an explanation of the contradictory findings in the literature.
\end{abstract}

The possible involvement of paradoxical sleep (PS) in learning and memory processes of animals has received increasing attention during the last few years. The importance of PS in the learning of new tasks has been investigated mainly by means of passive avoidance (Fishbein, 1970; Stern, 1970) and active avoidance techniques (Albert, Cicala, \& Siegel, 1970; Brill \& Goodman, 1969; Stern, 1970a, b; Joy \& Prinz, 1969). With the exception of the work of Stern $(1970,1971)$ and Fishbein (1970), the other studies (Albert et al, 1970; Brill \& Goodman, 1969; Joy \& Prinz, 1969) reported no effect of paradoxical sleep deprivation (PSD) on subsequent acquisition of shock-motivated behavior. Less use has been made of tasks employing positive reinforcement. Dement (1965) presented some preliminary findings on five PSD cats experiencing difficulty in learning a Y-maze with food reinforcement, whereas Stern (1970) reported that PSD impaired acquisition of a sugar water-motivated alternation discrimination.

The effects of PSD on the memory of pretrained tasks have also been evaluated by means of passive avoidance (Fishbein, 1971; Fishbein, McGaugh, \& Swartz, 1971; Pearlman \& Greenberg, 1969; Stern, 1970) and active avoidance techniques (Albert et al, 1970; Joy \& Prinz, 1969; Leconte \& Bloch, 1970; Stern, 1970; Wolfowitz \& Holdstock, 1971). The studies that employed a passive avoidance approach all implicated PS in memory consolidation. Fishbein (1971) actually distinguished between the effects of PSD on aspects of short- and long-term memory. However, with the exception of two studies (Leconte \& Bloch, 1970; Wolfowitz \& Holdstock, 1971), the work using conditioned avoidance techniques failed to support involvement of PS in memory consolidation. Wolfowitz and Holdstock (1971) used an approach similar to that employed by Fishbein (1971). Both groups of experimenters administered electroconvulsive shock (ECS) immediately after PSD

\footnotetext{
*This research was supported by a grant from the Human Sciences Research Council of South Africa.
}

and argued that the memory trace seemed to be held in a labile form during PSD. The one study that used a 6-unit T-maze with food reinforcement concluded that PSD prevented the integration of maze experiences acquired prior to PSD (Pearlman, 1970).

Since involvement of PS in learning and memory processes of animals has been studied almost exclusively by means of shock-motivated behavior, the present study investigated food-motivated behavior in a simple T-maze. It was hoped that this approach would shed some light on the conflicting evidence regarding involvement of PS in learning and memory processes.

\section{GENERAL METHOD}

\section{Subjects}

Seventy-two male hooded rats of the Long-Evans strain, weighing $285-455 \mathrm{~g}$ at the start of the experiment, were used. All animals were housed in constant illumination during the entire experiment.

\section{Apparatus}

The T-maze used is depicted in Fig. 1. The alleyways were $12.5 \mathrm{~cm}$ high, painted flat white, and covered with a Plexiglas top. Guillotine doors separated the startbox and the two arms from the main runway. As soon as all four feet of the rat were outside the startbox or inside one of the arms, the respective doors were lowered manually behind the animal to prevent retracing. A trial was terminated when the rat discovered the 0.045 -g Noyes food pellet.

\section{Procedure}

In order to facilitate discovery of the food pellet during maze learning, the animals received 10 food pellets in a glass cup that was used during training for 2 consecutive days in the home cage before being placed on a food-deprivation schedule. The food-deprivation schedule induced a $15 \%$ loss of body weight. After initially being deprived of food for $47 \mathrm{~h}$, the rats were maintained on a schedule of $23-\mathrm{h}$ deprivation for the remainder of the experiment. On the 12th day of deprivation, maze training started. During training, the animal was always reinforced in the maze arm opposite to the one preferred on the very first trial when no reinforcement was given. Each daily session consisted of 10 trials. Three minutes were allowed for a 




trial on the 1 st day and $1 \mathrm{~min}$ on the following sessions. At the end of each trial, the rat was manually returned to the startbox. On the basis of their performance during training, the animals were divided into three groups. One Group (Group SP) was deprived of FS by being placed on inverted flower pots with a base diameter of $6.5 \mathrm{~cm}$. In an attempt to control for dampness, restriction, and stress of the PSD procedure, Group SP was compared to animals maintained on flower pots with a base diameter of $11.5 \mathrm{~cm}$ (Group LP) and with rats living in their home cages (Group HC) (see Morden, Mitchell, \& Dement, 1967). The flower pots were kept in buckets containing approximately $7.0 \mathrm{~cm}$ of water. The animals on flower pots had daily breaks of an hour for feeding in their home cages. During this time, they were kept under observation in case they should fall asleep. Retention testing commenced after $72 \mathrm{~h}$ exposure to the flower pot living. Pilot studies established that PSD of more than $72 \mathrm{~h}$ superimposed upon a schedule of extended food deprivation posed a very severe strain on the animals. Each group was returned to its respective flower pot or home cage living quarter between post-PSD sessions.

\section{EXPERIMENT I}

Thirty-six rats were used in Experiment I. Each group consisted of 12 animals. The animals were trained towards a criterion of $70 \%$ or more correct responses on two consecutive sessions or for a maximum of four sessions before they were placed on the flower pots. In no case did the four rats that achieved the $70 \%$ criterion do so in less than three sessions. The reason for introducing PSD at such a relatively late stage of training was due to the fact that the majority of animals still functioned at little better than chance level after the third session, as is evidenced by the mean number of 5.9 correct trials. A considerable improvement in performance occurred on the fourth session. The 32 animals who had not reached criterion by then had a mean number of 7.3 correct trials. The groups were matched according to the percentage correct responses on the final training session and, where relevant, according to the number of sessions taken to achieve
$70 \%$ correct responses twice in succession. Two retention tests were administered. The first followed after $72 \mathrm{~h}$ PSD and the second after $96 \mathrm{~h}$ PSD.

\section{RESULTS}

No group differences were evident in the number of reinforced trials on the last session before PSD or on the two retention sessions after PSD (see Fig. 2). Combining the last session before PSD and the two postdeprivation sessions in a mixed analysis of variance (Myers, 1966) confirmed the absence of any group differences. Both the group main effect $[F(2,33)=0.08]$ and the Group by Session interaction $[F(4,66)=0.35]$ failed to approach significance. All groups tended to perform somewhat more poorly on the first session after deprivation but returned to predeprivation levels of performance by the second session (Fig. 2).

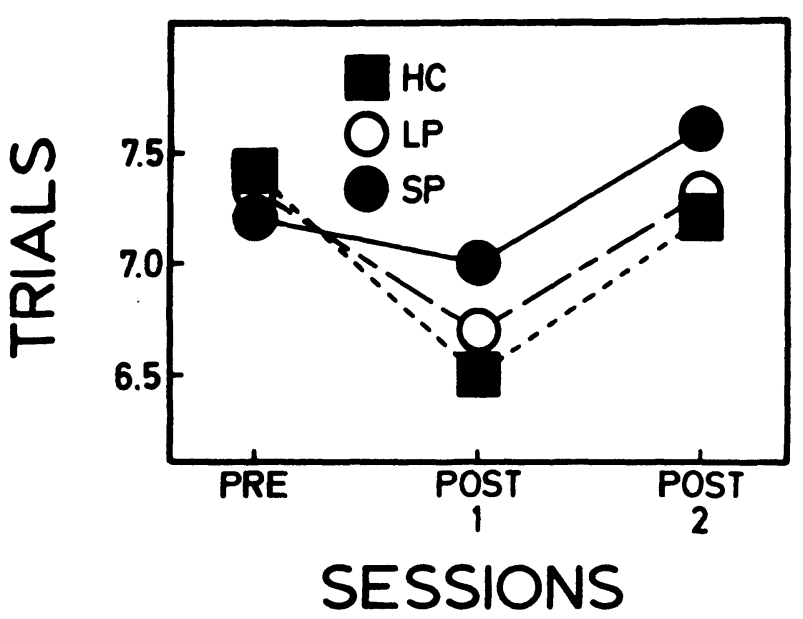

Fig. 2. Number of reinforced trials on the last training session and on two retention sessions. 
Since the additional training during postdeprivation sessions could have influenced the results, the first trial after PSD was analyzed separately. However, this analysis also failed to reveal any group differences. Nine animals of Group HC and eight each of Groups LP and $\mathrm{SP}$ chose the goal arm on the first retention trial.

\section{EXPERIMENT II}

Failure to find differences between the groups in Experiment I could have been due to the fact that PSD occurred after the animals had already experienced training sessions during which consolidation could have taken place, for sleep was not interfered with during training. In addition, the relatively easy nature of the retention task and the continued training during retention could also have contributed to the failure to find any differences between the groups. In Experiment II, an attempt was made to overcome these difficulties by using a reversal procedure carried out on a single session before initiation of PSD and to assess the effects of PSD by an extinction procedure.

Thirty-six animals were used in Experiment II. They received 10 trials until a criterion of $70 \%$ or more correct responses were obtained on 2 consecutive days. Animals not reaching criterion level by the 7 th day of training were rejected. Twenty-four hours after reaching criterion, reversal training was initiated. The arm opposite to that originally reinforced now became the positive side. Reversal training consisted of successive blocks of 10 trials with no delay between blocks or trials. One minute was allowed per trial, but the animals usually needed only a few seconds to run the maze. Reversal training continued until $70 \%$ or more correct responses were achieved in one set of 10 trials. Rats not reaching criterion by the fifth set of trials were to be rejected. Nine rats failed to meet criterion during training. Before being placed on flower pots, the groups were matched according to the number of days needed for the original training and number of trials required for reversal training. Each group consisted of nine animals. Retention testing began immediately after $72 \mathrm{~h}$ exposure on the flower pots and consisted of 20 extinction trials on each of 2 successive days. Trials were scored as correct if the arm reinforced during reversal training was entered.

\section{RESULTS}

The animals of all groups performed at chance level during both extinction sessions (Table 1). Subjecting the performance of the groups during the first and second half of both extinction sessions to a mixed design analysis of variance substantiated the lack of any group differences. Not only the group main effect $[F(2,24)=$ $0.82]$, but the Group by Trial $[F(2,24)=2.16]$ and Group by Session by Trial $[F(2,24)=0.01]$ interactions were decidedly insignificant. The direction chosen on
Table 1

Mean Number Correct Trials During Extinction

\begin{tabular}{|c|c|c|c|c|}
\hline \multirow[b]{2}{*}{ Group } & \multicolumn{2}{|c|}{ Extinction 1} & \multicolumn{2}{|c|}{ Extinction 2} \\
\hline & $\begin{array}{l}\text { Trials } \\
1-10 \\
\end{array}$ & $\begin{array}{c}\text { Trials } \\
11-20 \\
\end{array}$ & $\begin{array}{c}\text { Trials } \\
1-10\end{array}$ & $\begin{array}{l}\text { Trials } \\
11-20\end{array}$ \\
\hline $\mathrm{HC}$ & 5.0 & 5.5 & 4.7 & 5.3 \\
\hline LP & 4.9 & 4.5 & 4.2 & 3.9 \\
\hline SP & 4.7 & 4.8 & 5.2 & 5.3 \\
\hline
\end{tabular}

the first trial of reversal extinction also failed to differentiate between the groups. Three of the nine animals of Group SP and five each of Groups LP and HC chose the arm reinforced during reversal training.

\section{DISCUSSION}

The complete lack of any effect of PSD upon retention of tasks learned in a simple T-maze extends the contradictory nature of results obtained in studying PS involvement in memory to the area of appetitive tasks. The failure of PSD to impair retention of the two food-motivated tasks employed in the present experiment is in agreement with the majority of studies evaluating the effects of PSD on memory of active avoidance responses (Albert et al, 1970; Joy \& Prinz, 1969; Stern, 1970). On the other hand, studies that employed passive avoidance responses implicated PS in memory consolidation. The fact that PSD had no disruptive effects on the memory of tasks requiring responses of an active rather than an inhibitory nature suggests that the kind of task could be an important variable in evaluating the amnesic effects of PSD upon previously acquired tasks. Results obtained by studies evaluating the effects of PSD upon new learning seem to be in accordance with this idea. PSD disrupted acquisition of passive avoidance (Fishbein, 1970, 1971; Stern, 1970) but not active avoidance tasks (Albert, Cicala, \& Siegel, 1970; Brill \& Goodman, 1969; Joy \& Prinz, 1969). Although these results point to an interactive effect between PSD and the nature of the task to be acquired or retained, it must be kept in mind that Stern (1971) reported acquisition deficits following PSD that were not task specific. In addition, Pearlman (1970) found that PSD impaired retention of experiences in a complex maze. Thus, an explanation of the contradictory results in terms of the nature of the task can only be a qualified one at present.

In addition to the nature of the task, several aspects of the present experiment could have been responsible for the failure to demonstrate PSD effects on memory. In the first instance, it is possible that the 72 -h period of PSD represented too short a period to affect memory or even performance in the T-maze. However, this is unlikely, since Stern (1971) demonstrated disruptive effects on acquisition of a passive avoidance response following $72 \mathrm{~h}$ PSD. Secondly, the possibility exists of contamination of the effects of PSD on memory and 
food motivation. Increased food motivation, which is generally believed to be a consequence of PSD, may counteract whatever effects of amnesia there might be. Thirdly, the repeated training which the animals received in the maze during criterion training in Experiment I could have been responsible for a sufficient amount of consolidation to have occurred by the time PSD was initiated. This argument does not apply to Experiment II, however. In conjunction with this argument, it is possible that the effects of PSD on retention and learning may be much more subtle than was evaluated in the present experiment. In order to determine whether this is the case, it would be a good idea for future research to evaluate the effects of PSD on tasks differing in nature and complexity.

\section{REFERENCES}

Albert, J., Cicala, G. A., \& Siegel, J. The behavioral effects of REM sleep deprivation in rats. Psychophysiology, 1970, 6, $550-560$.

Brill, R. W., \& Goodman, I. J. Effects of REM sleep deprivation on memory in cats. Psychophysiology, 1969, 6, 226.

Dement, W. C. Recent studies on the biological role of rapid eye movement sleep. American Journal of Psychiatry, 1965, 122, 404-408.

Fishbein, W. Interference with conversion of memory from short-term to long-term storage by partial sleep deprivation. Communications in Behavioral Biology, Part A, 1970, 5, 171-175.

Fishbein, W. Dirsuptive effects of rapid eye movement sleep deprivation on long-term memory. Physiology \& Behavior,
$1971,6,279-282$.

Fishbein, W., McGaugh, J. I., \& Swarz, J. R. Retrograde amnesia: Electroconvulsive shock effects after termination of rapid eye movement sleep deprivation. Science, 1971, 172, 80-82.

Joy, R. M., \& Prinz, P. N. The effect of sleep altering environments upon the acquisition and retention of a conditioned avoidance response in the rat. Physiology \& Behavior, 1969, 4, 809-814.

Leconte, P., \& Bloch, V. Deficit de la retention d'un conditionnement après privation de sommeil paradoxal chez le rat. Centre de Recherche de l'Académie de Science (D) Paris, 1970, 271, 226-229.

Morden, B., Mitchell, G., \& Dement, W. Selective REM sleep deprivation and compensation phenomena in the rat. Brain Research, 1967, 5, 339-349.

Myers, J. L. Fundamentals of experimental design. Boston: Allyn \& Bacon, 1966.

Pearlman, C. A. Effect of rapid eye movement (dreaming) sleep deprivation on retention of avoidance learning in rats. U.S. Naval Submarine Medical Center Report No. 563, 1969.

Pearlman, C., \& Greenberg, R. Effect of REM deprivation on retention of avoidance learning in rats. Paper read at APSS, Denver, 1968.

Stern, W. C. Behavioral and biochemical aspects of rapid eye movement sleep deprivation in the rat. Dissertation Abstracts, 1970a, 31, 1574-1575.

Stern, W. C. The relationship between REM sleep and learning: Animal studies. International Psychiatry Clinics, 1970b, 7, 249-257.

Stern, W. C. Acquisition impairments following rapid eye movement sleep deprivation in rats. Physiology \& Behavior, 1971, 7, 345-352.

Wolfowitz, B. E., \& Holdstock, T. L. Paradoxical sleep deprivation and memory in rats. Communication in Behavioral Biology, 1971, 6, 281-284.

(Received for publication August 8, 1972; revision received September 16, 1972.) 